Mens

revue d'histoire intellectuelle de l'Amérique française

\title{
Des intellectuels en Europe et en Amérique. Un état de la question
}

\section{Pascale Ryan}

Volume 4, numéro 1, automne 2003

URI : https://id.erudit.org/iderudit/1024626ar

DOI : https://doi.org/10.7202/1024626ar

Aller au sommaire du numéro

Éditeur(s)

Centre de recherche en civilisation canadienne-française

ISSN

1492-8647 (imprimé)

1927-9299 (numérique)

Découvrir la revue

Citer cet article

Ryan, P. (2003). Des intellectuels en Europe et en Amérique. Un état de la question. Mens, 4(1), 9-37. https://doi.org/10.7202/1024626ar d'utilisation que vous pouvez consulter en ligne.

https://apropos.erudit.org/fr/usagers/politique-dutilisation/ 


\title{
DES INTELLECTUELS EN EUROPE ET EN AMÉRIQUE. UN ÉTAT DE LA QUESTION ${ }^{1}$
}

\author{
Pascale Ryan \\ Département des lettres et communications \\ Université de Sherbrooke
}

Les "intellectuels", comme groupe et comme notion, recèlent un paradoxe bistorique méconnu. Alors que le mot a moins d'un siècle d'usage courant, il est devenu un terme indispensable du discours politique, idéologique, sociologique, historique, voire psychologique. Même banalisé et usé par son abus de sens contradictoires, il nourrit encore les controverses savantes ou les essais à la mode qui, périodiquement, le relancent quand le paysage intellectuel devient trop morne. Comme concept, les "intellectuels » échappent ainsi au destin courant des vocables désignant un groupe social, celui d'une neutralisation progressive ou, au contraire, d'un ancrage bistorique daté.

Christophe Charle, Naissance des «intellectuels », 1880-1900.

Même après des années de recherches, il apparaît toujours difficile de concevoir la notion d'« intellectuel» autrement qu'à travers la figure emblématique de l'intellectuel de gauche contestataire que Jean-Paul Sartre a pu incarner dans 
la deuxième moitié du $\mathrm{XX}^{\mathrm{e}}$ siècle français. Il est d'ailleurs symptomatique de constater que, dans ce pays, le discours sur « la mort des intellectuels » coïncide, dans les années 1980, avec celle du philosophe existentialiste. Pourtant, dans les dernières années, plusieurs chercheurs ont permis de relativiser cette conception de l'intellectuel, qui a largement influencé l'historiographie québécoise. Ils font apparaitre les multiples identités possibles des intellectuels, largement tributaires des contextes nationaux et historiques. Une définition plus ouverte permet ainsi de comprendre l'intellectuel comme une figure changeante plutôt que figée, dans un processus évolutif d'une identité en construction. Ces travaux invitent à dresser un état de la question des intellectuels en Europe et en Amérique, démarche préalable à une relecture du cas canadien-français.

\section{L'intellectuel dreyfusard, une figure emblématique...}

On s'accorde à faire remonter l'origine de la figure moderne de l'intellectuel à l'Affaire Dreyfus ${ }^{2}$. Cette "affaire" cristallise en effet - au vu de l'historiographie française les caractéristiques " essentielles » - voire essentialistes de cet " homme du culturel mis en situation du politique ${ }^{3}$ » au $\mathrm{XX}^{\mathrm{e}}$ siècle. On assiste alors à un regroupement d'hommes de culture - écrivains, journalistes, universitaires - qui, en vertu d'un capital symbolique accumulé, prennent la parole collectivement et publiquement au nom de «la vérité », contre ce qui est perçu comme une injustice : la condamnation du capitaine Dreyfus pour haute trahison, alors que les preuves de sa culpabilité apparaissent trafiquées.

Trois phénomènes nouveaux distinguent les intellectuels dreyfusards de leurs ancêtres qui - tout au moins depuis le XVIII ${ }^{e}$ siècle - ont joué un rôle politique dans la société française, du philosophe et de «l'homme de lettres» du XVIII 


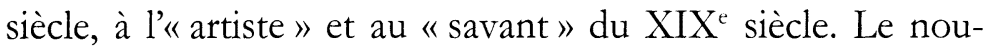
veau groupe social présente des caractéristiques nouvelles, dont l'autonomie face aux institutions et à la hiérarchie est sans doute la plus importante.

L'examen de la pétition en faveur du capitaine Dreyfus révèle également une nouvelle manière d'intervenir dans le champ du pouvoir en introduisant une rupture dans les règles du combat public qui distingue les dreyfusards de leurs «ancêtres ${ }^{4}$ ». Ils revendiquent le droit au scandale, en appuyant l'article diffamatoire d'Émile Zola "J'accuse»; le droit de se liguer pour donner plus de force à la protestation; et enfin le droit de revendiquer un pouvoir symbolique tiré de l'accumulation des titres que la plupart des signataires mentionnent à la suite de leur nom. Si la revendication de ces trois droits n'est pas nouvelle, c'est leur conjugaison et la signification attribuée à des moyens anciens qui provoquent une rupture.

C'est également à ce moment qu'est popularisé le substantif «intellectuel». Terme à connotation péjorative auparavant, plutôt utilisé sous la forme de l'adjectif, il désigne une intellectualité excessive, un romantisme malsain ${ }^{5}$. Appliqué par dérision aux dreyfusards, il est alors revendiqué par ceuxci pour s'autodésigner face à leurs adversaires. En plus de ses sens déjà usités, le substantif prend alors une coloration nouvelle qui s'imposera dorénavant dans le vocabulaire courant.

L'intellectuel de type dreyfusard qui émerge à ce moment devient dès lors une figure emblématique de la vie intellectuelle et politique française dans le "siècle des intellectuels ${ }^{6}{ }^{\prime}$, marqué par une intense activité de ceux-ci dans le débat public, plus particulièrement au moment des crises nationales qui divisent la société française? Les acteurs euxmêmes ont dû se définir par rapport à ce modèle entre "le devoir d'engagement », personnifié par Jean-Paul Sartre et «le devoir de réserve ", revendiqué par Raymond Aron. 
L'étude des intellectuels comme groupe social telle qu'elle s'est développée en France depuis les années 1950 tant chez les historiens que chez les sociologues - s'est articulée autour de ce moment de rupture que constitue l'Affaire Dreyfus. C'est dans le cadre d'une histoire politique en quête de renouveau que les historiens ont cherché à comprendre les liens qu'entretiennent les intellectuels avec la politique ${ }^{8}$. Cette démarche, amorcée par René Rémond dans les années 1950, a provoqué une "explosion bibliographique » dans les années 1980 et $1990^{\circ}$. À l'aide d'outils conceptuels - l'étude des trajectoires individuelles et collectives, des générations intellectuelles et des réseaux de sociabilité - les chercheurs ont voulu comprendre les grands axes d'engagement de ces « hommes du culturel mis en situation du politique » en France au $\mathrm{XX}^{\mathrm{e}}$ siècle ${ }^{10}$. Ils ont pratiqué une histoire contextuelle classique où les origines sociales et l'itinéraire personnel de l'intellectuel - considéré ici comme un sujet relativement libre-, replacé dans son époque, éclairent les raisons de l'engagement sans le surdéterminer.

Parallèlement s'est développée chez les sociologues groupés autour de Pierre Bourdieu une approche principalement axée sur une analyse des stratégies de pouvoir et de légitimation dans le champ intellectuel. L'intellectuel - producteur, consommateur et manipulateur de biens symboliques n'est plus considéré ici comme un individu libre mais bien comme un produit social dont il convient d'étudier les conditions de production et d'intervention dans le champ culturel.

Les deux approches - concurrentes parfois - ont grandement contribué à éclairer l'histoire des intellectuels comme groupe social en France. Centrées le plus souvent sur le cadre spatio-temporel français de la fin du XIX ${ }^{e}$ siècle et sur le $\mathrm{XX}^{e}$, elles ont cependant également contribué à faire de l'intellec- 
tuel qui émerge au moment de l'Affaire Dreyfus une « exception française ».

\section{Remise en question...}

Au terme de vingt années de travaux en histoire des intellectuels en France, certains chercheurs en sont venus à questionner la pertinence d'un champ disciplinaire consacré spécifiquement à l'histoire des intellectuels. Christophe Charle s'est élevé à plusieurs reprises contre la sectorisation des disciplines qui freine une histoire globale des intellectuels, seule susceptible d'éclairer leurs différentes identités possibles.

La chronologie « intellectuelle » commence également à être remise en question. Michel Trebitsch mettait récemment en garde contre cet «a priori initial que l'intellectuel commence en 1897 et qu'il doit être en train de mourir ces temps-ci ». Il suggère de "sans cesse replacer leur mode d'intervention dans la Cité, dans une histoire qu'on peut presque faire remonter au Moyen Âge ${ }^{11}$.» Il est d'ailleurs maintenant bien établi que des intellectuels - ou leurs ancêtres, sous d'autres vocables - ont, bien avant 1897 , joué un rôle politique, ce qu'une concentration des études sur le $\mathrm{XX}^{\mathrm{e}}$ siècle avait pu masquer ${ }^{12}$. L'élargissement du cadre spatio-temporel d'analyse qu'a pratiqué Christophe Charle montre par ailleurs que l'intellectuel n'est pas une spécificité française et qu'il ne se réduit pas à sa version dreyfusarde ${ }^{13}$.

La quête d'une définition "définitive » de l'intellectuel - qui a tant hanté les chercheurs qui se sont attelés à la tâche de cerner cette catégorie sociale aux contours flous a trop souvent mené à une réduction unidimensionnelle et « essentialiste » du personnage, devenu souvent objet fétiche ${ }^{14}$. L'approche comparatiste pratiquée dans les dernières années a permis justement de remettre en question les a priori initiaux, de confronter les grilles d'analyses étrangères au mo- 
dèle français et de prendre en compte les réflexions méthodologiques et théoriques auparavant ignorées par les chercheurs hexagonaux ${ }^{15}$. La contribution de Christophe Charle a été particulièrement marquante dans cette démarche. Selon ce chercheur, les trois registres - social, culturel et politique - auxquels réfère la notion d'intellectuel - , lorsqu'ils sont dissociés, sont trop limitatifs pour arriver à bien saisir le personnage. Ainsi, l'approche sociologique, privilégiant la notion de «travailleurs intellectuels », tend à étendre indéfiniment l'ensemble des groupes désignés à mesure que s'élève le niveau culturel d'un pays. Les définitions de type culturaliste - d'où la dimension sociologique est absente - mènent à une perspective élitiste où les chercheurs se concentrent sur les créateurs les plus en vue, " promus au rang d'exemples ou de porteparole de l'ensemble des intellectuels ». Enfin, une approche purement politique

masque le fait que l'espace d'action politique et symbolique des intellectuels dépend étroitement des transformations des sociétés globales, des champs intellectuels (nationaux, infranationaux ou transnationaux) et de la concurrence d'autres formes possibles d'action politique $^{16}$.

Érigées en définition standard, ces approches deviennent anhistoriques, privilégiant «abusivement l'un des trois registres ou rabattant l'une ou l'autre des dimensions sur la troisième ». Le recours à la combinaison des trois niveaux d'analyse s'avère donc nécessaire, et surtout fertile, comme l'a montré Charle dans son étude sur les intellectuels européens au XIX ${ }^{e}$ siècle.

Dans une synthèse récente, Michel Leymarie faisait le point sur les intellectuels et la politique en France. Signe des temps, bien que cette étude se concentre sur le $\mathrm{XX}^{\mathrm{e}}$ siècle, la généalogie intellectuelle est clairement retracée, tout au moins 
jusqu'au Moyen Âge. L'auteur adopte une approche extensive des intellectuels, proche de celle de Christophe Charle, tenant compte des divers registres associés au substantif. De l'Affaire Dreyfus à la fin du XX $\mathrm{XX}^{\mathrm{e}}$ siècle, les intellectuels français sont présentés ici sous leurs multiples identités. Leur soidisant silence dans la société française des années 1980, alors qu'ils se retirent du champ social, apparaît en fait comme un processus de mutation identitaire plutôt que comme le signe d'une disparition. L'activité intellectuelle s'est professionnalisée, la légitimation et la consécration proviennent désormais non plus des pairs, mais du grand public et de l'audimat. On assiste à l'émergence de nouvelles figures concurrentes. Les pétitions contre les lois Debré-Pasqua (1997) sont particulièrement révélatrices de ce phénomène : ce sont « des femmes et des hommes des médias, réalisateurs ou comédiens, intellectuels d'un nouveau type, [qui] tiennent la place qu'occupaient écrivains ou universitaires il y a cent ans ${ }^{17}$ ». Cette métamorphose "procède d'abord de la démocratisation de la société et de l'extension de la fonction qui était - ou aurait dû être, constamment - celle des "clercs", comme les nommait Julien Benda ${ }^{18} »$. Aux confins du XX $\mathrm{XX}^{\mathrm{e}}$ siècle français, la figure de l'intellectuel «se dilate et se délite».

Une définition plus ouverte permet ainsi de comprendre l'intellectuel comme une figure changeante plutôt que figée, d'en retrouver les multiples identités possibles dans un processus évolutif d'une identité en construction. Expert engagé dans les débats de sa cité, l'intellectuel doit nécessairement prendre ses racines dans sa société d'origine ou d'appartenance. Or cette société est elle-même en constante évolution. L'étude du personnage et de sa fonction exige donc une nécessaire démarche d'historicisation et de contextualisation. 


\section{Les intellectuels en Europe}

Cette mise au point conceptuelle était nécessaire. La prégnance du modèle français de l'intellectuel dreyfusard a en effet eu ses répercussions sur les historiographies extrahexagonales en Europe. Les historiens étrangers qui ont repris le questionnaire français dans leur propre contexte national se sont trouvés confrontés à plusieurs difficultés. On ne retrouve pas, dans la plupart de ces pays, d'actes fondateurs du genre de l'Affaire Dreyfus. La compétence politique reconnue aux intellectuels en France est absente de la plupart des autres pays. La question du lexique ne se pose pas non plus dans les mêmes termes. Le substantif apparaît tout d'abord en France et semble s'exporter dans la plupart des pays européens, francophones ou non, à la suite de l'Affaire. Il est adopté plus ou moins rapidement selon qu'il existe ou non, dans la langue de réception, un terme fondé sur d'autres racines ou historiquement plus daté pour désigner la même réalité que le néologisme français ${ }^{19}$. Christophe Charle note tout de même que, dès la phase d'émergence et de diffusion du substantif, les nouvelles représentations sociales auxquelles il réfère comportent maintenant les trois registres, social, culturel et - dans une moindre mesure - politique, registres «partiellement ou totalement absents de la sémantique des terminologies antérieures plus limitées ${ }^{20} »$.

Le cas de l'Angleterre est sans doute représentatif. Le substantif n'y est pas utilisé avant les années 1870 , et le développement de l'usage anglais a été largement influencé par les développements en français, en allemand et en russe. Au $\mathrm{XIX}^{\mathrm{e}}$ siècle, le terme est plutôt utilisé comme adjectif et décrit des compétences mentales et culturelles. L'usage du substantif se répand avec la publicité donnée à l'Affaire Dreyfus et est adopté pour désigner le nouveau groupe créé par l'avancement de la science, la réforme des universités et les répon- 
ses des gens éduqués face à la montée de la société industrielle. Il exprime la nouvelle relation de cette élite culturelle à la société en général. L'usage du substantif demeure cependant peu courant, mais l'idée même à laquelle il convie n'est pas inconnue au XIX ${ }^{\mathrm{e}}$ siècle. La question du rôle social des intellectuels a été longuement discutée par Coleridge, Mill et Arnold, entre autres. L'adoption du néologisme - qui en est venu à remplacer le «clerisy » élaboré par Coleridge - n'est, selon Peter Allen, qu'une variation moderne d'un concept déjà bien établi au XIX ${ }^{e}$ siècle $^{21}$.

Le rapport des intellectuels au politique dans les pays européens varie selon le type de vie politique qu'on y trouve. Les différentes études de cas montrent clairement que cette variable est essentielle dans le rôle que les intellectuels se donnent dans leur société d'appartenance. Par exemple, en Angleterre, contrairement à la France, les élites politiques n'ont pas besoin des intellectuels, et ces derniers n'éprouvent aucune gêne à être associés au pouvoir. Les extrêmes intellectuels y sont absents. Les intellectuels en Angleterre ont le plus souvent été les gardiens du consensus, fixant eux-mêmes les bornes au legitimate dissent, à l'intérieur duquel prennent place les débats, plus ou moins actifs selon les périodes ${ }^{22}$. Le cas des «Public moralists » du tournant du $\mathrm{XX}^{\mathrm{e}}$ siècle montre cependant que s'ils conçoivent différemment leur fonction dans la société, ils présentent plusieurs des caractéristiques des intellectuels français. Experts dans leur domaine respectif, c'est au nom de valeurs morales qu'ils s'engagent dans les débats de société. Ils recourent aux mêmes stratégies : utilisation de leurs réseaux de sociabilité, des revues et des journaux afin de diffuser leurs idées et de convaincre leurs contemporain ${ }^{23}$.

Divisée linguistiquement et culturellement, la Suisse est également un pays où la vie politique est basée sur le consen- 
sus. L'intellectuel au sens français du terme ne correspond pas à une figure sociale helvétique ${ }^{24}$. Les élites intellectuelles - notamment les historiens - ont cependant régulièrement pris position sur la question jurassienne. Claude Hauser retrace trois figures de l'intellectuel dans ce débat. Ce sont sans doute les intellectuels séparatistes qui correspondent le mieux au modèle dreyfusard. Ils revendiquent le substantif pour se désigner, croient à la force des idées et s'assignent un rôle de guide dans la prise de conscience du peuple jurassien. Ils n'hésitent pas à s'engager sur le terrain du politique au service de la cause. Du côté antiséparatiste, les intellectuels se perçoivent comme des "médiateurs" entre les cultures. Enfin, un certain nombre d'intellectuels jurassiens, tout en refusant de prendre parti, revendiquent leur condition en tant que défenseurs d'une identité jurassienne spécifique, basée sur l'appartenance à la culture française et l'expérience d'une histoire commune. Ils assument une condition élitiste tout en étant autant que possible dégagés des débats politiques ${ }^{25}$.

Autre cas de figure d'un pays à l'identité nationale faible, la Belgique, divisée linguistiquement, religieusement et politiquement, ne possède pas non plus un milieu intellectuel autonome et unifiée ${ }^{26}$. Le pays est petit, l'édition, faible et la presse, concentrée depuis 1945. Le champ culturel n'a qu'une autonomie restreinte. Paris joue le rôle de centre et la Belgique francophone, de périphérie. Le corps social fait peu appel aux intellectuels autonomes, sauf lorsque les piliers ont besoin de légitimer leur position. De plus, l'usage du substantif est loin d'être courant et le milieu intellectuel semble particulièrement dénué de toute conscience de groupe $\mathrm{e}^{27}$. L'engagement individuel et collectif apparait toujours comme un phénomène marginal, et sa participation politique s'inscrit à l'intérieur des clivages belges: catholiques/non catholiques, droite/gauche, flamands/francophones. Lorsque les intellec- 
tuels sont présents sur la scène politique, c'est à titre d'experts. Selon Bradfer, la vie politique belge, au-delà de ses clivages idéologiques et confessionnels, est caractérisée par son pragmatisme traditionnel et sa méfiance à l'égard des considérations trop théoriques. Ce sont moins les idées que les intérêts qui la gouvernent.

Pourtant, en étudiant le champ littéraire belge et les rapports de force qui le divisent - divisions entre un «art social » à vocation utilitaire et un « art socialisé »-, Paul Aron met au jour les engagements de ces écrivains au tournant du $\mathrm{XX}^{\mathrm{e}}$ siècle ${ }^{28}$. À ce moment de l'histoire belge, les écrivains socialistes jouent le rôle d'intellectuels, s'engageant au nom d'un capital symbolique dans les causes auxquelles ils adhèrent.

En Allemagne, comme ailleurs, l'histoire politique a fortement marqué la nature de l'intervention des intellectuels dans le débat public. Les historiens s'y sont principalement concentrés sur l'étude du groupe social qui compose le Bildungsbürgertum (les professeurs d'université) ${ }^{29}$. Issus de l'émergence de l'université allemande au $\mathrm{XIX}^{\mathrm{e}}$ siècle, ces professeurs se perçoivent sous la République de Weimar comme les gardiens de la culture allemande face à une modernité menaçante et envahissante ${ }^{30}$. Ils refusent la modernité technique, politique et culturelle et sympathisent avec l'idéologie et le régime nazi, perçu comme le gardien des anciennes valeurs. Ils ont ainsi contribué à légitimer le nouveau pouvoir en place à partir de 1933, avec les conséquences que l'on sait.

Il est frappant de constater que la plupart des chercheurs qui se sont penchés sur l'étude des intellectuels dans leur pays respectif hésitent à leur attribuer le substantif. Ils arrivent pourtant à dégager leur contribution à la vie de la cité. Le problème réside sans doute dans l'adhésion au modèle français. Comme le fait remarquer Hans-Manfred Bock, « du point 
de vue de la sociologie politique, on peut considérer qu'il n'y a pas de société moderne de type européen où la fonction de l'intellectuel n'existe pas, même si elle s'exerce sous des dénominations et dans des cadres différents ${ }^{31} »$.

S'interrogeant sur le fait que les intellectuels britanniques ne correspondent pas à la norme française, Stefan Collini suggère que les comparaisons peuvent être "more opentextured, and hence revealing, if each case is recognised as but one possibility on a spectrum of possibilities ${ }^{32} »$. C'est également ce qu'avance Christophe Charle. L'approche comparative de cet auteur révèle que le discours du groupe englobé par le bildungsbürgertum - que les historiens allemands ont eu tendance à voir comme unique et typiquement allemand - est en fait également partagé par les intellectuels dominants français au moment de l'Affaire Dreyfus. Il s'agirait d'une des identités possibles des intellectuels comme groupe fermé face à un champ intellectuel en pleine transformation dans les deux pays ${ }^{33}$.

Cette approche se révèle beaucoup plus fertile, comme l'a montré Charle dans son étude des intellectuels en Europe au XIX ${ }^{e}$ siècle. Le recours aux trois registres auxquels réfère la notion de l'intellectuel lui permet de dégager un tableau d'ensemble de l'évolution des différentes figures de l'intellectuel européen au XIX ${ }^{e}$, qui mèneront à l'émergence de l'intellectuel moderne à la fin de la période. Tout en éclairant les spécificités nationales des intellectuels dans chacun des pays étudiés, l'auteur fait ressortir des cycles et des rythmes communs, des influences réciproques et des transferts entre traditions nationales.

\section{Les intellectuels en Amérique}

Les études sur les intellectuels dans différents pays d'Amérique dégagent un modèle proche de l'intellectuel or- 
ganique de Gramsci ${ }^{34}$. Dans ces pays jeunes, aux identités nationales en développement, ces intellectuels - en général experts ou journalistes - ont comme projet de société la construction d'un imaginaire et d'une culture propres à leur pays. Cette construction est le plus souvent basée sur un nationalisme en quête d'identité.

À New York, par exemple, les intellectuels - désignés par Thomas Bender par le terme générique "intellect» qui désigne la vie intellectuelle plutôt que les acteurs eux-mêmes $^{35}$ - ont participé à la construction d'une culture urbaine dans un milieu neuf et cosmopolite, loin des modèles européens en qui, pourtant, les intellectuels cherchent à la fois un modèle et un repoussoir. Bender étudie le rôle des individus - avocats, journalistes, professeurs - et des institutions Églises, universités, clubs, journaux - dans l'émergence de l'espace public aux $\mathrm{XIX}^{\mathrm{e}}$ et $\mathrm{XX}^{\mathrm{e}}$ siècles. Ce n'est que dans les années 1940 et 1950 que la vie intellectuelle new-yorkaise arrive à maturité, au terme d'un long processus de construction, avec l'immigration massive d'intellectuels européens pendant la guerre, donnant à la métropole américaine sa dimension de capitale culturelle internationale.

Les intellectuels au Mexique et au Brésil partagent plusieurs caractéristiques. Depuis l'Indépendance, ils participent à la construction imaginaire d'une nation. Ils en sont les idéologues, les médiateurs et les artisans. Au Mexique, la Révolution les a aspirés dans la fonction publique, rendant la vie intellectuelle étroitement subordonnée au régime politique, concentrée sur la résolution des grands problèmes nationaux. Le nationalisme, leur plus grande construction culturelle, a servi à renforcer leurs liens avec le régime et à légitimer ce dernier $^{36}$. Dans une perspective française, la mise en service de leurs talents et de leur capital symbolique au service de l'État n'en fait pas des intellectuels mais bien des experts. 
L'absence d'opposition légale et la diversité idéologique limitée au sein du pays, un champ littéraire et culturel fortement dépendant de l'État, l'absence de critique et de positionnement de ces experts face au régime constituent un handicap à la formation de l'esprit critique et de la fonction contestataire des intellectuels. Ils jouent cependant le rôle d'intellectuels organiques, dans une perspective gramscienne.

Au Brésil, les intellectuels se sont eux-mêmes définis comme formant un secteur spécifique ayant vocation collective à intervenir dans le domaine politique - dont ils cherchent à énoncer les fondements - à partir de leurs compétences. Ils définissent leur responsabilité par rapport à la nation, à l'État et au peuple ${ }^{37}$. Ils partagent la croyance selon laquelle le politique ne peut être séparé de la science, et la science du politique. Cette figure est sans doute plus autonome que celle de l'intellectuel mexicain. Elle est cependant proche de l'intellectuel organique.

Ces différentes études de cas montrent une variété d'identités possibles de l'intellectuel, en fonction du rôle qu'ils sont appelés à jouer dans leur société d'origine.

\section{Les intellectuels au Québec}

Au Québec, jusqu'aux années 1980 du moins, c'est à partir d'une problématique fort différente qu'on s'est le plus souvent intéressé aux intellectuels. Cherchant à comprendre le "retard " économique, social et politique des Canadiens français au XIX ${ }^{e}$ siècle et dans la première moitié du $\mathrm{XX}^{e}$ siècle, les premiers analystes en ont attribué « la faute» au poids d'une idéologie monolithique de conservation ${ }^{38}$. Une étude trop rapide du discours de l'élite intellectuelle, tel qu'il se présentait dans ses revues et ses journaux, les a fait conclure à un décalage entre cette idéologie et la réalité concrète de la majorité des Canadiens français ${ }^{39}$. C'est ce décalage qui aurait 
été à la source du retard constaté. Avec le recul, ces travaux pionniers prennent souvent l'allure d'un procès. Au banc des accusés : ces élites intellectuelles traditionalistes qui se sont prononcées sur la place publique sur le sort de la collectivité canadienne-française. Ces travaux ont été longuement discutés dans les dernières années par de nouvelles générations de chercheurs qui en ont souligné les faiblesses méthodologiques, les a priori idéologiques et la connaissance incomplète des idéologies en place ${ }^{40}$.

Comme le souligne Catherine Pomeyrols, il est sans doute temps d'entreprendre une histoire des intellectuels québécois «qui ne soit pas seulement une étude des idéologies, qui ne se confonde ni avec une histoire des "grands hommes" ni avec leurs conceptions de la réalitée ${ }^{41} »$. En effet, si les travaux de Pierre Trépanier et de ses étudiants sur les droites intellectuelles au Québec ont grandement contribué à une meilleure compréhension globale des idéologies avant 1960, ce milieu intellectuel reste somme toute peu connu. On s'est le plus souvent intéressé aux intellectuels "modernisateurs", ceux qu'on a associés à la période d'ébullition des années 1950 et à la Révolution tranquille, laissant dans l'ombre les «traditionalistes ${ }^{42} »$. Comment pourrait-on en effet être un intellectuel lorsqu'on est de droite, nationaliste et catholique de surcroît ? Comme le souligne Xavier Gélinas, on a longtemps douté de la pertinence de la contribution de la droite aux débats précédant la Révolution tranquille:

[...] passé une certaine époque, et surtout après 1960, on ne pouvait penser qu'à gauche. Méritent-ils le titre d'intellectuels, ces gens peu nombreux, coupés des idées vivantes ? Ces esprits sclérosés qui soutiennent l'Union nationale, au pouvoir puis dans l'opposition, ne se discréditent-ils pas d'avance ? $^{43}$ 
Pourtant, ils ont joué un rôle dans la quête nationaliste et la modernisation du Québec des années 1950 et $1960^{44}$. Leurs idéaux n'ont d'ailleurs pas toujours été divergents de ceux des intellectuels de "gauche». Par exemple, la revue catholique et nationaliste Tradition et Progrès - fondée en 1957 pour faire un contrepoids à ce qui est perçu comme un dévoiement du nationalisme canadien-français par le "gauchisme "-, se rapproche tantôt des "clérico-conservateurs »- comme sur la question du rôle temporel de l'Église - tantôt des néonationalistes en ce qui concerne le rôle de l'État. Elle oscille constamment entre les deux pôles incarnés dans son titre, comme le montre Xavier Gélinas.

Pour Catherine Pomeyrols, la définition réductrice de l'intellectuel engagé de gauche de type sartrien a conduit à cette occultation, particulièrement notable en ce qui concerne l'entre-deux-guerres. Le modèle français aurait été utilisé pour définir les intellectuels assimilés aux forces progressistes avant la Révolution tranquille. L'intellectuel existait déjà, soutientelle, mais ne correspondait pas toujours à cette définition ${ }^{45}$. Bien qu'à l'aide du questionnaire français, elle introduise de nouvelles perspectives dans l'analyse des intellectuels québécois de l'entre-deux-guerres, le résultat est mitigé. Une mise en contexte déficiente, où la formation idéologique découle d'une transposition des idéologies de droite françaises, nuit à l'apport le plus original de son étude, l'analyse des collèges classiques comme matrices idéologiques de générations d'intellectuels au Québec. Les vingt-trois individus choisis à l'aide des dictionnaires biographiques font partie des plus connus de la période. Le traitement qu'elle en fait n'apporte pas un éclairage nouveau. On s'étonne par ailleurs des absences comme celle de Paul Bouchard -, pas toujours justifiées. Une méconnaissance des sources provoque des erreurs factuelles 
qui, intégrées à son analyse, nuisent à la crédibilité de ses conclusions $^{46}$.

Adoptant un point de vue opposé - à partir de la même définition de l'intellectuel élaborée par Pascal Ory -, Yvan Lamonde soutient au contraire qu'on ne trouve pas d'intellectuels au Québec avant les années $1930^{47}$. C'est à partir d'une approche lexicale - entre autres - qu'il arrive à cette conclusion ${ }^{48}$. Bien que les conditions d'émergence de «l'intellectuel » soient présentes dès le dernier quart du XIX ${ }^{\mathrm{e}}$ siècle au Québec, le fait que le substantif n'ait pas le même sens qu'en France et qu'il ne soit ni utilisé ni revendiqué par les intellectuels eux-mêmes constitue un obstacle à la possibilité de l'intellectuel québécois avant $1900^{49}$. Par peur de l'anachronisme, l'auteur récuse donc l'utilisation d'un concept pour une période antérieure à son apparition et ce, malgré l'usage qu'en ont fait des chercheurs français tel Jacques Le Goff pour le Moyen Âge ou Daniel Roche pour l'époque moderne.

Lamonde soulève par ailleurs d'autres objections pour ce qui concerne le XIX ${ }^{\mathrm{e}}$ siècle. La prégnance de l'ultramontanisme - qui bloque l'individualité - empêche la possibilité d'un intellectuel laïque. L'absence d'État laïque ou républicain freine l'autonomie, la faculté critique et la possibilité de rupture face à l'ordre dominant, qualités propres à l'intellectuel français - même catholique - à la même époque. Le milieu intellectuel ne dispose pas d'une masse critique suffisante pour être autonome et s'opposer collectivement à la censure cléricale de la presse et des institutions et associations. Le contrôle clérical du système d'éducation empêche l'émergence d'« une communauté de gens de savoir plus large que celle des clercs et des professions libérales, ces derniers s'investissant par ailleurs beaucoup dans la politique ${ }^{50} »$. Cet engagement dans la politique partisane empêche selon lui la distanciation, l'autonomie et l'esprit critique essentiels à l'intellectuel. 
Du début du $\mathrm{XX}^{\mathrm{e}}$ siècle jusqu'aux années 1930, Lamonde perçoit cependant quatre facteurs de progrès dans le processus d'émergence des intellectuels au sens où il les entend ${ }^{51}$. Il constate tout d'abord un éloignement de la politique partisane, notant au passage le rôle qu'a joué l'abbé Groulx dans ce transfert vers l'« homme du culturel mis en situation $d u$ politique et non de la politique ». Deuxième signe, cette volonté de faire place au savoir en dehors des professions libérales ou du journalisme. Des individus comme Léon Gérin et Errol Bouchette tentent de construire ce savoir à l'extérieur d'une université émergente. Par la suite, une tradition de science susceptible de concurrencer une «doctrine » y est inaugurée par Édouard Montpetit, Esdras Minville, MarieVictorin et Jacques Rousseau. La consolidation de l'université qui s'ensuit vient s'ajouter aux deux autres facteurs. Les experts, savants et universitaires, de plus en plus nombreux, se constituent en catégorie sociologique et en masse critique, rendant possible, «au fil de la vie publique, la catégorie politique de l'homme d'idées qui prend position sur les affaires publiques $^{52}$ ». Enfin, l'entrée en scène des "modernistes» minoritaires, qui contestent l'orthodoxie nationaliste et régionaliste, revendiquant les droits de l'individu créateur et la liberté dans les thèmes de création, permet la liberté intellectuelle. Dans un article plus récent, analysant l'émergence de l'intellectuel suisse, nationaliste et catholique au tournant du $\mathrm{XX}^{\mathrm{e}}$ siècle, Lamonde y voit des convergences avec le cas canadien-français qui lui permettent de remettre en question le modèle dreyfusard comme point de départ de l'émergence de l'intellectuel d'ici au siècle passé $e^{53}$.

Depuis les travaux pionniers d'Yvan Lamonde, plusieurs chercheurs, à la fin des années 1990, se sont détachés de la recherche de l'intellectuel dreyfusard. Bernard Andrès, par exemple, retrouve, dans la génération de la Conquête, la pre- 
mière « intelligentsia » de l'histoire québécoise. Les bouleversements sociopolitiques, idéologiques et littéraires qui prennent place à la fin du XVIII ${ }^{e}$ siècle confrontent les Canadiens à un tout nouveau type d'altérité. Ils doivent se définir une identité propre et choisir s'ils désirent passer du statut de « sujets » à celui de " citoyens ». Les premiers débats littéraires, philosophiques et constitutionnels apparaissent alors. De ces débats émerge un individu qui n'est "plus seulement l'"Homme de lettres" ou le "Philosophe" mais déjà "l'homme de culture [...] mis en situation du politique" ", qui recourt à tous les modes d'intervention publique recensés par Lamonde pour la fin du XIX ${ }^{e}$ siècle. Cette " génération d'écriture » contribue à la création d'un tout nouvel espace public. Pour Andrès, ces individus sont donc des intellectuels, ne serait-ce que "pour le simple fait d'avoir pensé au Québec, d'avoir pensé le Québec au terme du XVIII ${ }^{\mathrm{e}}$ siècle $^{54} »$. Fernande Roy a montré, pour le $\mathrm{XIX}^{\mathrm{e}}$ siècle, le rôle d'intellectuels qu'ont joué les journalistes dans la création d'un espace public, la conquête de la liberté d'expression et la poursuite des idéaux démocratiques $^{55}$. Yves Gingras a par ailleurs mis au jour la contribution de Marie-Victorin et du groupe de recherche qu'il dirigeait aux débats sur l'enseignement secondaire et supérieur. Ceux-ci ont pris la parole au nom d'une certaine vision de la société, celle d'une société nord-américaine, urbaine et industrialisée, qui nécessite des institutions adaptées à ses besoins $^{56}$. Marie-Victorin n'apparaît plus, dans cette analyse, comme un franc-tireur, mais bien comme le porte-parole d'un groupe beaucoup plus large, qu'on a eu tendance à ignorer à cause de la concentration des analyses sur la question nationale.

Les réflexions de ces trois derniers auteurs montrent qu'il faut reposer la question des intellectuels au Québec en d'autres termes. En effet, l'identité des intellectuels n'est ni 
statique ni unidimensionnelle. La recherche de la figure emblématique de gauche, laïcisée, autonome et contestataire a masqué les autres formes qu'ils ont pu revêtir à différentes époques et dans différents contextes. $\mathrm{Ni}$ " catégorie socioprofessionnelle » ni « personnage irréductible ${ }^{57}$ », l'intellectuel se définit par cette fonction qu'il s'attribue de penser la société et de proposer le résultat de sa réflexion sur la place publique. Comme le suggère Fernande Roy, la véritable question est la suivante : qui, à une époque donnée, dans un contexte donné, joue ce rôle d'intellectuel ? La réponse à une telle question nécessite d'historiciser la fonction des intellectuels et leurs conditions d'émergence à différentes époques. Le recours aux registres culturel, social et politique tel que l'a pratiqué Christophe Charle apparaît comme le meilleur moyen permettant d'historiciser la figure de l'intellectuel et ses modalités d'intervention dans la cité. Ses multiples identités peuvent s'incarner dans différentes figures successives - dans un processus évolutif d'une identité en construction - ou encore concurrentes, dans un contexte où les débats sociaux polarisent l'ensemble de la société, comme au moment de l'Affaire Dreyfus. Selon la formule de Michel Leymarie, les « intellectuels sont peut-être — et d'abord - les échos sonores de leur époque $^{58}$ ». Leur identité découle directement de ces débats dans lesquels ils s'engagent. Le cas des scientifiques québécois est exemplaire, comme le souligne Yves Gingras :

[...] la construction d'un espace relativement autonome pour la pratique de la recherche scientifique passait par la promotion dans l'espace public d'une certaine conception de la société civile. Une fois ce travail accompli, les scientifiques ont pu se retrancher dans leurs laboratoires et vaquer à leurs occupations dans leur nouvelle tour d'ivoire ${ }^{59}$. 
Il existait donc, bien avant les années 1930, des « hommes du culturel mis en situation du politique » au Canada français. Ni individus isolés ni "francs-tireurs", ils ont, depuis la fin du XVIII ${ }^{\mathrm{e}}$ siècle, joué un rôle dans la construction d'un espace public, la quête de libertés individuelles et des idéaux démocratiques. Profondément engagés dans la vie de leur cité — d'un engagement qui relève du politique —, ils ont lancé pétitions, manifestes et journaux de combat. Ils ont développé une sociabilité intellectuelle et politique à travers la fondation de clubs, d'associations et d'instituts - tel l'Institut canadien - et se sont, quelquefois, lancés dans la politique afin de promouvoir leurs idéaux. Ils ont bien souvent dû affronter la censure politique et cléricale, à laquelle ils se sont opposés, au risque parfois de leur propre liberté personnelle. Dans une synthèse récente, Yvan Lamonde rappelait l'intensité de ces combats qui ont marqué les $\mathrm{XVIII}^{\mathrm{e}}$ et $\mathrm{XIX}^{\mathrm{e}}$ siècles canadiens-français ${ }^{60}$. Tout comme la lutte pour l'autonomisation du champ intellectuel, qui lui est postérieure, cette lutte pour les libertés fondamentales confère aux acteurs un statut d'intellectuel, comme l'a observé Christophe Charle pour le XIX ${ }^{\mathrm{e}}$ siècle européen ${ }^{61}$.

Si ces premiers intellectuels canadiens-français, leurs discours et leurs combats ont fait l'objet de nombreuses études, c'est surtout à travers le prisme de l'idéologie qu'ils ont été analysés ${ }^{62}$. Bernard Andrès et Fernande Roy ont cependant posé les jalons d'une relecture de ces deux siècles à partir de la problématique des intellectuels telle qu'elle est articulée ici. D'autres études empiriques s'imposent pour ces périodes. Ces deux chercheurs ont cependant permis d'identifier des identités possibles de l'intellectuel engagé dans la cité au Canada français. 
En ce qui concerne le premier $\mathrm{XX}^{\mathrm{e}}$ siècle, l'accent mis sur les idéologies, et notamment sur la question nationale, a pu biaiser les perspectives, l'engagement nationaliste étant pour certains, un obstacle à l'émergence de l'intellectuel, pour d'autres, un écran masquant les autres milieux intellectuels. De plus, comme le souligne Yves Gingras, l'insistance sur quelques figures de proue bien connues, considérés trop souvent comme des individus isolés et non comme faisant partie de groupes dont ils pouvaient être les porte-parole n'a pas été jusqu'à maintenant très éclairante. C'est cependant la démarche la plus souvent adoptée par ceux qui se sont penchés sur la question. Il apparait donc impératif de reprendre la question sur une base plus large, à partir de nouvelles sources et de nouveaux acteurs. Les pistes de recherche sont nombreuses. Par exemple, ces nouveaux et nombreux lieux de militantisme qui émergent au tournant du $\mathrm{XX}^{\mathrm{e}}$ siècle et qui se distinguent de ceux du siècle précédent par leur refus de la politique partisane. Ces mouvements sont loin d'être apolitiques. Ils relèvent directement d'une conception citoyenne de l'action intellectuelle qui cherche à influencer les instances décisionnelles par l'action militante et par la propagande. En tant que membres de l'élite intellectuelle, leurs militants, clercs et laïcs, perçoivent comme un devoir civique l'engagement sur la place publique au nom de leurs conceptions du bien-être collectif des Canadiens français ${ }^{63}$. L'exemple de la Ligue d'action française, appelée à devenir dans les années 1930, la Ligue d'action nationale, en est un exemple frappant ${ }^{64}$. Les nombreux journaux et revues intellectuels fondés durant cette période sont également des pépinières d'intellectuels qu'il conviendrait d'étudier à partir de la problématique soulevée ici. Nombreux sont les milieux intellectuels d'avant la Révolution tranquille qui attendent leurs chercheurs... 


\section{NOTES}

${ }^{1}$ Cette étude, tirée de mes travaux de doctorat, a bénéficié de l'appui financier du Prêt d'honneur de la Société Saint-Jean-Baptiste, du FCAR et de la Fondation PAFARC de l'UQAM.

${ }^{2}$ L’Affaire Dreyfus a été abondamment discutée par les historiens français. Je ne traiterai pas ici l'ensemble de cette historiographie. Voir, entre autres, Pascal Ory et Jean-François Sirinelli. Les intellectuels de l'Affaire Dreyfus à nos jours. Paris, Armand Colin, 1986. 263 p. ; Christophe Charle. Naissance des « intellectuels », 1880-1900. Paris, Éditions de Minuit, 1990. 272 p.

${ }^{3}$ Pascal Ory, «Qu'est-ce qu'un intellectuel ? ", dans Pascal Ory, dir. Dernières questions aux intellectuels, Paris, Olivier Orban, 1990, p. 24.

${ }^{+}$Christophe Charle, op. cit., p. 8.

${ }^{5}$ Geneviève Idt. «L'intellectuel avant l'affaire Dreyfus ».Cabiers de lexicologie, 15 (1969), pp. 35-46.

${ }^{6}$ Michel Winock. Le siècle des intellectuels. Paris, Seuil, 1999. 696 p.

${ }^{7}$ Jean-François Sirinelli. Intellectuels et passions françaises. Manifestes et pétitions au $X X^{r}$ siècle. Paris, Fayard, 1990. 365 p.

${ }^{8}$ Voir entre autres, l'article pionnier de René Rémond, « Les intellectuels et la politique ",. Revue française de science politique, vol. 9, n" 4 (décembre 1959), pp. 860-880. Pour un aperçu des différents travaux qui ont suivi l'article programmatique de Rémond, voir Jean-François Sirinelli. « Les intellectuels », dans René Rémond, dir. Pour une histoire politique, Paris, Seuil, 1996 [1988], pp. 199-231.

'Louis Bodin, Les intellectuels existent-ils ?, Paris, Bayard Éditions, 1997, p. 9.

"I Sur les notions de trajectoires, de générations et de réseaux, voir JeanFrançois Sirinelli. «Le hasard ou la nécessité ? Une histoire en chantier : l'histoire des intellectuels ». Vingtième siècle, 9 (janvier-mars 1986), pp. 97-108; du même auteur, "Les intellectuels ", dans Pour une histoire politique, loc. cit., ainsi que l'introduction à sa thèse Génération intellectuelle. Khâgneux et Normaliens dans l'entre-deux-guerres, Paris, Quadrige/PUF, 1994, [1988], pp. 10-13. Ces questions ont également été étudiées par l'équipe de l'Institut d'histoire du temps présent, Générations intellectuelles. Cahier 6, Paris, CNRS, novembre 1987. $104 \mathrm{p}$. 
"Michel Trebitsch, «Devons-nous définir l'intellectuel ? », dans Manon Brunet et Pierre Lanthier, dir. Linscription sociale de lintellectuel, Québec, Les Presses de l'Université Laval/L'Harmattan, 2000, p. 39.

${ }^{12}$ Jean-François Sirinelli, Intellectuels et passions françaises, op. cit., p. 21 ; Christophe Charle. Les intellectuels en Europe au XIX' siècle. Paris, Seuil, 1996. 379 p. ; Daniel Roche. Les Républicains des lettres. Gens de culture et Lumières au XV TII siècle. Paris, Fayard, 1988. 393 p. ; Jacques Le Goff,.I es intellectuels an Moyen Age. Paris, Seuil. 224 p. (Collection « Points-Histoire)».

${ }^{13}$ Christophe Charle, op. cit.

${ }^{1+}$ Michel Trebitsch, loc. cit., p. 39.

${ }^{15}$ Marie-Christine Granjon. « Une enquête comparée sur l'histoire des intellectuels : synthèse et perspectives ", dans Michel Trebitsch et Marie-Christine Granjon, dir. Pour une bistoire comparée des intellectuels, Paris, Éditions complexes, 1998, pp. 19-36.

${ }^{16}$ Christophe Charle, op. cit., pp. 18-19.

${ }^{17}$ Michel Leymarie, Les intellectuels et la politique en France, Paris, PUF, 2001, p. 117. (Collection "Que sais-je ?»).

${ }^{18}$ Ibid., p. 121.

${ }^{19}$ Christophe Charle, op. cit., p. 245.

21" Christophe Charle, "L'histoire comparée des intellectuels en Europe ", dans Michel Trebitsch et Marie-Christine Granjon, dir. Pour une bistoire comparée des intellectuels, Paris, Éditions complexes, 1998, p. 43.

${ }^{21}$ Peter Allen, "The Meaning of "an Intellectual" in Nineteenth- and Twentieth-Century English Usage ", University of Toronto Quarterly, vol. 55 (1986), p. 346.

${ }^{22}$ François-Charles Mougel. "Les intellectuels britanniques », dans MarieChristine Granjon, Nicole Racine et Michel Trebitsch, dir. Histoire comparée des intellectuels. Pré-actes du colloque Histoire comparée des intellectuels tenu à Paris le 23 janvier 1997, Paris, CNRS/IHTP, 1997, pp. 69-74.

${ }^{23}$ Stefan Collini, Public Moralists. Political Thought and Intellectual Life in Britain. 1850-1930, Oxford, Clarendon Press, 1991, pp. 45-68.

${ }^{24}$ Brigitte Studer. «Jalons pour l'étude du cas suisse », dans Marie-Christine Granjon, Nicole Racine et Michel Trebitsch, dir. Histoire comparée des intellectuels, op. cit., pp. 31-36. 
${ }^{25}$ Claude Hauser. «Les intellectuels et la question jurassienne », dans MarieChristine Granjon, Nicole Racine et Michel Trebitsch, dir. Histoire comparée des intellectuels, op. cit., pp. 23-30.

${ }^{26}$ Paul Aron et José Gotovitch. «Situation des intellectuels en Belgique », dans Marie-Christine Granjon, Nicole Racine et Michel Trebitsch, dir. Histoire comparée des intellectuels, op. cit., pp. 11-18.

${ }^{27}$ Philippe Bradfer. « Quelques remarques sur les intellectuels en Belgique », dans Marie-Christine Granjon, Nicole Racine et Michel Trebitsch, dir. Histoire comparée des intellectuels, op. cit., 1997, pp. 9-22.

${ }^{28}$ Paul Aron. Les écrivains belges et le socialisme (1880-1913). Bruxelles, Labor, $1985.278 \mathrm{p}$.

${ }^{29}$ Christophe Charle attribue ce phénomène à la segmentation de la recherche dans ce pays entre différentes disciplines. Les travaux des littéraires sur les écrivains et les journalistes ont souligné leur importance dans le débat public, où ils jouent un véritable rôle d'intellectuels. «Intellectuels, Bildungsbürgertum et professions au XIX ${ }^{\mathrm{e}}$ siècle. Essai de bilan historiographique comparé (France, Allemagne) ». Actes de la recherche en sciences sociales, $\mathrm{n}^{\text {os }}$ 106-107 (mars 1995), pp. 85-95.

311 Fritz K. Ringer, The Decline of the German Mandarins. The German Academic Community, 1890-1933, Hanover et Londres, University Press of New England, 1990 [1969], p. viii.

${ }^{31}$ Hans Manfred Bock, "Quelques remarques sur les intellectuels en Allemagne et sur leur historiographie », dans Marie-Christine Granjon, Nicole Racine et Michel Trebitsch, dir. Histoire comparée des intellectuels, op. cit., p. 37.

${ }^{32}$ Stefan Collini, "Intellectuals in Britain and France in the TwentiethCentury », dans Marie-Christine Granjon, Nicole Racine et Michel Trebitsch, dir. Histoire comparée des intellectuels, op. cit., p. 57.

${ }^{33}$ Christophe Charle, loc. cit.

${ }^{34}$ Antonio Gramsci. «La formation des intellectuels », dans François Ricci, dir. Gramsci dans le texte, Paris, Éditions sociales, 1975, pp. 597-608.

${ }^{35}$ Selon cet auteur, le substantif « intellectuel » apparaît aux États-Unis trois mois après l'Affaire Dreyfus, mais n'est incorporé dans l'usage qu'en 1907. Ce sont les "Young intellectuals» de Greenwich Village, en rupture avec les générations intellectuelles précédentes qui revendiquent le terme pour la première fois. Thomas Bender. New York Intellect. A History of Intellectual Life in 
New York City, from 1750 to the Beginnings of Our Own Time. New York, Alfred A. Knopf, 1987. 422 p.

${ }^{36}$ Annick Lempérière, "Les intellectuels mexicains au $\mathrm{XX}^{\mathrm{e}}$ siècle. Origines historiques et devenir d'un modèle », dans Marie-Christine Granjon, Nicole Racine et Michel Trebitsch, dir. Histoire comparée des intellectuels, op. cit., p. 248. Voir également du même auteur, Intellectuels, États et société au Mexique : les clercs de la nation, 1910-1968. Paris, L'Harmattan, 1992. 393 p.

${ }^{37}$ Daniel Pécaut. « Réflexions sur les relations entre les intellectuels et la politique au Brésil ", dans Marie-Christine Granjon, Nicole Racine et Michel Trebitsch, dir. Histoire comparée des intellectuels, op. cit., pp. 209-223.

${ }^{38}$ Des travaux plus récents en histoire économique, sociale et politique ont permis de relativiser ce « retard » du Québec par rapport au monde occidental en général et à l'Amérique du Nord en particulier. Ce n'est cependant pas mon propos d'en discuter ici. À ce sujet, voir la synthèse de Paul-André Linteau, René Durocher et Jean-Claude Robert. Histoire du Québec contemporain. Montréal, Boréal, 1989. 2 volumes.

${ }^{39}$ Voir entre autres Michel Brunet. "Trois dominantes de la pensée canadienne-française : l'agriculturisme, l'anti-étatisme et le messianisme », dans La présence anglaise et les Canadiens; études sur l'bistoire de la pensée des deux Canadas, Montréal, Beauchemin, 1958, pp. 113-166 ; Maurice Tremblay. «Orientations de la pensée sociale ", dans Jean-Charles Falardeau, dir. Essais sur le Québec contemporain, Québec, PUL, 1953, pp. 193-215 ; Pierre Elliot Trudeau. "La province de Québec au moment de la grève de l'amiante », dans Pierre Elliot Trudeau, dir. La grève de l'amiante, Montréal, Éditions Cité Libre, pp. 191. Les travaux rassemblés par Fernand Dumont dans les différents volumes des Idéologies au Canada francais. Québec, PUL, 1974-1981, quoique plus nuancés, participent généralement de cette tendance.

${ }^{40}$ À ce sujet, voir les travaux de Fernande Roy, Progrès, harmonie, liberté. Le libéralisme des milieux d'affaires francophones à Montréal au tournant du siècle, Montréal, Boréal, 1988, pp. 11-43, Xavier Gélinas. « La droite intellectuelle et la Révolution tranquille : le cas de la revue Tradition et Progrès, 1957-1962». Canadian Historical Review, vol. 77, n 3 (septembre 1996), pp. 353-369 ; JeanClaude Dupuis. Nationalisme et catholicisme. L'Action française de Montréal (19171928). Thèse de maitrise (histoire), Université de Montréal, 1992. $329 \mathrm{f}$.

${ }^{41}$ Catherine Pomeyrols, Les intellectuels québécois : formation et engagements, 1919. 1939, Paris, L'Harmattan, 1996, p. 108. 
${ }^{42}$ C'est, entre autres, le cas de Léon Dion qui, bien qu'il n'ignore pas complètement les traditionalistes, met l'accent sur les «modernisateurs » dans son livre Québec 1945-2000, Les intellectuels et le temps de Duplessis. Sainte-Foy, PUL, $1993.452 \mathrm{p}$.

${ }^{43}$ Xavier Gélinas, loc. cit., p. 355.

${ }^{44}$ Pierre Trépanier, « Notes pour une histoire des droites intellectuelles canadiennes-françaises à travers leurs principaux représentants (1770-1970) », Les Cabiers des Dix, 48 (1992), p. 163.

${ }^{45}$ Catherine Pomeyrols, op. cit.

46 Par exemple, on est étonné d'apprendre à la page 459 que Rodolphe Dubé a écrit dans L'Action française. Le pseudonyme de François Hertel a bien été utilisé par un collaborateur, mais il s'agit de $\mathrm{M}^{\mathrm{gr}}$ Georges Courchesne. Voir Lionel Groulx, Mes Mémoires, Montréal, Fides, 1971, II : 131. D'autre part, Rodrigue Villeneuve a bien collaboré à cette revue, mais il n'a jamais été membre de la ligue du même nom (p. 378). Par ailleurs, Harry Bernard n'a fondé ni la Ligue ni la revue L'Action nationale. (pp. 253, 264). Cette erreur factuelle est d'autant plus surprenante que l'auteur a pourtant consulté Les étapes d'une carrière (causeries autobiographiques et textes connexes) (édité par François-Albert Angers), Montréal, Les Presses HEC/Fides, 1988, p. 81.

${ }^{47}$ Yvan Lamonde. "Les "intellectuels" francophones au Québec au XIX siècle : questions préalables ». RHAF, vol. 48, n" 2 (automne 1994), pp. 153185 ; «L'Affaire Dreyfus et les conditions d'émergence de l'intellectuel vues des Amériques ", dans Michel Trebitsch et Marie-Christine Granjon, dir. Pour une histoire comparée des intellectuels, Paris, Éditions complexes, 1998, pp. 111123 ; « L'époque des francs-tireurs : les intellectuels au Québec, 1900-1930», dans Manon Brunet et Pierre Lanthier, dir. L'inscription sociale de lintellectuel, op. cit., pp. 189-211, et « Problèmes et plaisir de la biographie ». RHAF, vol. 54, n"1 (été 2000), pp. 89-94.

${ }^{48}$ Lamonde s'inspire du travail de Geneviève Idt, loc. cit., sur l'apparition du substantif en France au moment de l'Affaire Dreyfus. Ce chercheur adopte la même démarche, retraçant l'occurrence du substantif au Québec en 1901 seulement.

${ }^{49}$ Bernard Andrès et Catherine Pomeyrols se sont livrés au même travail pour les XVIII et $\mathrm{XX}^{e}$ siècles. Bernard Andrès. "Y a-t-il un intellectuel dans le Siècle ? ou Penser au Québec à la fin du XVIII" siècle », dans Manon Brunet et Pierre Lanthier, dir. L'inscription sociale de l'intellectuel, op. cit., pp. 43-60. Catherine Pomeyrols, op. cit. Pour cette dernière, le fait que le substantif soit peu 
utilisé pourrait venir du fait qu'il est associé aux intellectuels dreyfusards, dans lesquels les intellectuels québécois ne se reconnaissent pas.

${ }^{50}$ Yvan Lamonde, «Les "intellectuels" francophones au Québec au XIX siècle : questions préalables », loc. cit., p. 177.

${ }^{51}$ Yvan Lamonde, «L'époque des francs-tireurs : les intellectuels au Québec, 1900-1930», loc. cit.

${ }^{52}$ Ibid., p. 207.

${ }^{53}$ «L'Alpe et la vallée laurentienne : les intellectuels et le nationalisme en Suisse romande et au Québec au tournant du XX $\mathrm{XX}^{\mathrm{e}}$ siècle », dans Claude Hauser et Yvan Lamonde, dir. Regards croisés entre le Jura, la Suisse romande et le Québec, Québec, Presses de l'Université Laval/L'Harmattan, 2002, pp. 317 333. Dans cet article fort intéressant, Lamonde analyse les dénominateurs communs qui permettent de mieux comprendre que la France, la Suisse et le Québec partagent des questions similaires sur le plan social et intellectuel. Cette analyse laisse entrevoir un certain nombre de pistes de recherche en histoire comparative intellectuelle entre la Suisse romande et le Québec, pistes beaucoup plus intéressantes que celles soulevées par Catherine Pomeyrols, qui réduit le Québec à une zone périphérique culturelle de la France.

${ }^{54}$ Ibid., p. 60.

${ }^{55}$ Fernande Roy. «Les intellectuels canadiens-français du siècle dernier : les journalistes ", dans Manon Brunet et Pierre Lanthier, dir. L'inscription sociale de lintellectuel, op. cit., pp. 341-350.

${ }^{56}$ Yves Gingras. «Le rôle d'intellectuel des scientifiques québécois », dans Manon Brunet et Pierre Lanthier, dir. L'inscription sociale de l'intellectuel, op. cit., pp. 331-340.

${ }^{57}$ Pascal Ory, loc. cit., p. 27.

${ }^{58}$ Michel Leymarie, op. cit., p. 121.

${ }^{59}$ Yves Gingras, loc. cit., p. 337.

(11) Yvan Lamonde. Histoire sociale des idées au Québec, 1760-1896. Montréal, Fides, 2000. 574 p.

${ }^{61}$ Christophe Charle, Les intellectuels en Europe au XIX' siècle, op. cit., p. 26.

${ }^{62} \mathrm{Je}$ renvoie à l'étude de Fernande Roy pour une critique de ces travaux. Voir Progrès, harmonie, Liberté, op. cit. Exception notable, la biographie d'Errol 
Bouchette par Alain Lacombe, Errol Bouchette, 1862-1912. Un intellectuel. Montréal, Fides, 1997. 236 p.

${ }^{63}$ Comme le fait remarquer Yves Gingras, le choix d'exclure les religieux du champ intellectuel semble relever d'une confusion entre la catégorie sociologique d'intellectuel et l'usage du substantif et de l'épithète par les acteurs sociaux. Yves Gingras, loc. cit., p. 339. Dans une thèse récente, Marie-Pier Luneau montrait d'ailleurs clairement un Groulx écrivain autonome face à l'Église dans ses stratégies de légitimation et de consécration littéraires. À l'aide de la sociologie de la littérature, cette chercheure a analysé tous les manuscrits de Groulx et sa correspondance avec plus d'une quinzaine d'interlocuteurs littéraires. On attend d'autres travaux de ce genre. Marie-Pier Luneau. Le manche et la cognée : Lionel Groulx, une vie d'écritures. Thèse de doctorat (lettres et communications), Université de Sherbrooke, 2001. 486 f.

${ }^{64}$ Pascale Ryan. Des intellectuels dans la Cité. La Ligue d'action nationale, $1933-$ 1960. thèse de doctorat (histoire), Université du Québec à Montréal, 2002. $315 \mathrm{f}$. 